\title{
Spectrum Trading in Cognitive Radio Networks Using Multistage Bayesian Game
}

\author{
Feng-Tsun Chien*, Ronald Y. Chang ${ }^{\dagger}$, and Yu-Wei Chan ${ }^{\ddagger}$ \\ * Department of Electronics Engineering, National Chiao Tung University, Taiwan \\ ${ }^{\dagger}$ Research Center for Information Technology Innovation, Academia Sinica, Taiwan \\ ‡Department of Information Management, Chung Chou University of Science and Technology, Taiwan \\ Email: ftchien@mail.nctu.edu.tw, rchang@citi.sinica.edu.tw, and ywchan@dragon.ccut.edu.tw
}

\begin{abstract}
In this paper, we study spectrum trading in cognitive radio (CR) networks with multiple primary services (PSs) and multiple secondary services (SSs) from a game-theoretic perspective. We propose a multistage Bayesian game-based trading model which accounts for unknown private information of players (for example, the number of user connections in PSs may be unknown to the SSs) as in practical network scenarios. The perfect Bayesian equilibrium (PBE) is derived by solving an involved sequential optimization problem. We formulate the joint Karush-Kuhn-Tucker (KKT) conditions and use the KKT translation technique to obtain the PBE at each stage. Simulation demonstrates the convergence of the sequence of strategies in the multistage Bayesian game.
\end{abstract}

\section{INTRODUCTION}

Cognitive radio (CR) has been considered as a promising technique to improve the efficiency of radio spectrum utilization. One critical challenge in CR, however, is how to incentivize the licensed primary services (PS) to share their unused spectrum with unlicensed secondary services (SS) or users. As mentioned in [1], leasing available spectrum to unlicensed services is an attractive option that provides an incentive for licensed operators to support the deployment of CRs. The action of leasing provides licensed operators with monetary profits while fulfilling the requirements of unlicensed services. Since most licensed and unlicensed services are rational and selfish decision makers interacting with one another, game theory arises as a useful tool to analyze the micro-economics and design the decision strategies for CR networks.

An overview of the general idea and recent developments about dynamic spectrum sharing games can be found in [2]. $\mathrm{Ji}$ and Liu [3] considered the spectrum auction mechanism for CR networks with multiple primary and multiple secondary users, and discussed the competitive equilibrium, cheating behaviors, and prevention measures for collusion. Niyato and Hossain [4] proposed utility functions that incorporate both monetary gain and quality-of-service $(\mathrm{QoS})$ satisfaction of wireless services for spectrum sharing games. Feng et al. [5] proposed a hybrid pricing framework and modeled the strategic choices of the SUs as a noncooperative game. Yin et al. [6] proposed a two-stage spectrum trading market (longterm and short-term) and modeled long-term and short-term

This work was supported in part by the Ministry of Science and Technology, Taiwan under Grants MOST 103-2221-E-001-027 and 103-2218-E-009-033. markets by contract theory and Stackelberg game, respectively. Lin et al. [7] proposed a flexible auction mechanism in the spectrum trading market with wireless service providers and spectrum holders.

The aforementioned work assumed that each player in the modeled game has complete knowledge about the other players' private information. To account for unknown private information at each player, it is useful to consider a Bayesian or stochastic game to study the behaviors of spectrum trading in a sequential/dynamic manner [8], [9]. In [8], the dynamics of the spectrum access strategies under a stochastic game framework were characterized, and the learning/prediction of future dynamics was studied based on the learning theory. In [9], the spectrum market was modeled as a four-stage Bayesian game, and it was shown that by incorporating the insurance mechanism into spectrum trading, the spectrum efficiency can be improved.

In this work, we consider that each player (PS or SS) in the game has its own private information unknown to other players in a CR network with multiple PSs and multiple SSs. The contributions of this paper are summarized as follows.

- We model spectrum trading in a multi-PS multi-SS CR network with the multistage Bayesian game. The considered game accommodates unknown private information of players (e.g. the number of user connections in PSs, which may be unknown to the SSs), as in practical network scenarios. Each PS can offer different prices per unit bandwidth to SSs, and SSs with different QoS can demand different bandwidth sizes from each PS.

- We obtain the PBE using a unique approach inspired by the backward induction principle, in which the KarushKuhn-Tucker (KKT) conditions of all SSs are translated into PSs' constraints and an active-set based algorithm is used to solve the involved sequential optimization problem. We verify that joint KKT conditions lead to a feasible solution. We also numerically study the convergence of the sequence of strategies in the multistage Bayesian game.

The outline of this paper is as follows. Sec. II describes the problem and preliminaries. Sec. III presents a general formulation of the multistage Bayesian game for spectrum trading in a multi-PS multi-SS CR network. Sec. IV presents simulation results. Conclusion is drawn in Sec. V. 


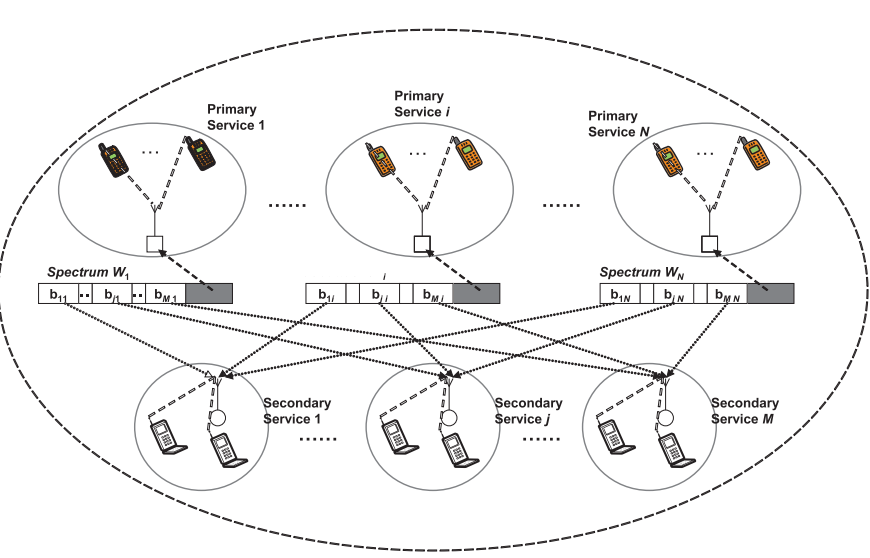

Fig. 1. A cognitive radio network with $N$ primary services and $M$ secondary services.

\section{Multistage Bayesian Game for Spectrum TRADING}

\section{A. Problem Description}

We consider the spectrum trading problem in a cognitive radio network with $N$ legitimate primary services (e.g. the existing cellular services) and $M$ secondary services (e.g. small networks with CR base stations and CR users), as shown in Fig. 1. The $i$ th PS operates on its own available and exclusive spectrum $W_{i}$. In the spectrum trading market, PSs compete in leasing their spectrum to the SSs to maximize their profits, while SSs buy the spectrum opportunities from the PSs based on the offered prices. We consider that each PS or SS is rational and selfish. Furthermore, to reflect a practical scenario, we consider that each PS or SS has its own private information, which is referred to as type in the literature, unknown to other PSs or SSs (e.g. the number of local connections within each service, the channel quality, etc.).

\section{B. Game Formulation}

We model the spectrum trading problem with private information as a multistage Bayesian game. The evolution of a repeated multistage game is illustrated in Fig. 2, where one unit time period comprises two stages. In the first stage, the $i$ th PS sets price $p_{j i}$ per unit bandwidth to the $j$ th SS, and in the second stage, the $j$ th SS requests bandwidth $b_{j i}$ from the $i$ th PS, for all $i$ and $j$. All PSs and SSs are the players of the game, in which the PSs serve as the leaders and SSs the followers in the game. To deal with the unknown private information in each player, we incorporate Bayesian updating process in the game which allows each player to update its posterior beliefs (i.e. posterior probabilities) about other players' private information by observing their actions in prior stages. Each player acts in the current stage according to the updated beliefs, which will be described in Sec. III.

A multistage Bayesian game $\Gamma$ at stage $t$ is defined by

$$
\Gamma=\left\langle\mathcal{I}, \mathcal{A}_{x}\left(h^{t}\right), \theta_{x}, \mathcal{P}_{x}, \mu_{x}\left(\boldsymbol{\theta}_{-x} \mid \theta_{x}, h^{t}\right): x \in \mathcal{I}\right\rangle .
$$

We describe each element in $\Gamma$ as follows. $\mathcal{I} \triangleq \mathcal{I}_{l} \cup \mathcal{I}_{s}$ is the set of players with $\mathcal{I}_{l}=\left\{l_{1}, \ldots, l_{N}\right\}$ being the set of

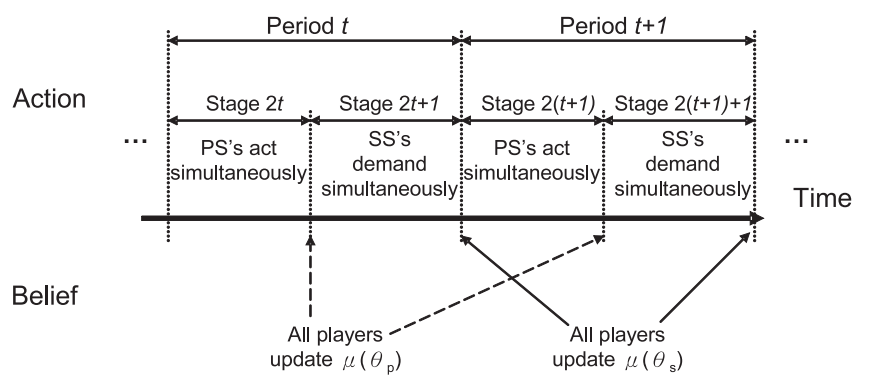

Fig. 2. The time evolution of the proposed multistage Bayesian game.

all legitimate PSs and $\mathcal{I}_{s}=\left\{s_{1}, \ldots, s_{M}\right\}$ being the set of all SSs. $\mathcal{A}_{x}\left(h^{t}\right)$ is the set of actions available for player $x$ given a history $h^{t}=\left(\mathbf{a}^{0}, \ldots, \mathbf{a}^{t-1}\right)$ at the beginning of stage $t$, where $\mathbf{a}^{\tau}=\left(\mathbf{a}_{l_{1}}^{\tau} \ldots \mathbf{a}_{l_{N}}^{\tau}, \mathbf{a}_{s_{1}}^{\tau} \ldots \mathbf{a}_{s_{M}}^{\tau}\right)$ is the action profile consisting of the actions from all players (including PSs and SSs) at stage $\tau$, with $\mathbf{a}_{l_{i}}^{\tau} \in \mathcal{A}_{l_{i}}\left(h^{\tau}\right)$ and $\mathbf{a}_{s_{j}}^{\tau} \in \mathcal{A}_{s_{j}}\left(h^{\tau}\right)$ being the action of the $i$ th PS and the $j$ th SS at stage $\tau$, respectively. The type profiles of PSs and SSs are denoted by $\boldsymbol{\theta}_{l}=\left(\theta_{l_{1}}, \ldots, \theta_{l_{N}}\right)$ and $\boldsymbol{\theta}_{s}=\left(\theta_{s_{1}}, \ldots, \theta_{s_{M}}\right)$, respectively. In particular, we write $\boldsymbol{\theta}_{-l_{i}}\left(\boldsymbol{\theta}_{-s_{j}}\right)$ to represent the type profile $\boldsymbol{\theta}_{l}\left(\boldsymbol{\theta}_{s}\right)$ excluding $\theta_{l_{i}}\left(\theta_{s_{j}}\right)$. The overall type profile is $\boldsymbol{\theta}=\left(\boldsymbol{\theta}_{s}, \boldsymbol{\theta}_{l}\right)$. The actual type value for player $x$ is denoted by $\widehat{\theta}_{x}$, and the actual type profile for PSs, SSs, and the overall players are $\widehat{\boldsymbol{\theta}}_{l}, \widehat{\boldsymbol{\theta}}_{s}$, and $\widehat{\boldsymbol{\theta}}$, respectively. $\mathcal{P}_{x}$ represents the profit function (i.e. the net utility) of player $x$, which is a mapping from the space $\mathcal{H}^{\tau} \times \boldsymbol{\theta}$ to the set of real numbers $\mathbb{R}$, where the set $\mathcal{H}^{t}$ contains all possible histories $h^{t}$ 's at time $t$, with $\mathcal{H}^{0}$ being the null set. $\mu_{x}\left(\boldsymbol{\theta}_{-x} \mid \theta_{x}, h^{t}\right)$ is player $x$ 's belief about other players' types given its type $\theta_{x}$ and the history $h^{t}$. In this paper, unlike a static game of incomplete information, we update the beliefs about other players' types stage-by-stage. Each player's action depends on the latest update of beliefs.

Define $\mathbf{p}_{i}^{l, \tau}=\left(p_{1 i}^{\tau}, \ldots, p_{M i}^{\tau}\right)^{T}, \mathbf{p}_{j}^{s, \tau}=\left(p_{j 1}^{\tau}, \ldots, p_{j N}^{\tau}\right)^{T}$, $\mathbf{b}_{i}^{l, \tau}=\left(b_{1 i}^{\tau}, \ldots, b_{M i}^{\tau}\right)^{T}$, and $\mathbf{b}_{j}^{s, \tau}=\left(b_{j 1}^{\tau}, \ldots, b_{j N}^{\tau}\right)^{T}$. In each time period, the $i$ th PS sets price $\mathbf{a}_{l_{i}}^{\tau}=\mathbf{p}_{i}^{l, \tau}$ at the even stage and is inactive at the odd stage. On the other hand, the $j$ th SS is inactive at the even stage and demands $\mathbf{a}_{s_{j}}^{\tau}=\mathbf{b}_{j}^{s, \tau}$ bandwidth at the odd stage.

\section{Solution Concept and Belief Update}

The solution concept in a multistage Bayesian game with incomplete information is called the perfect Bayesian equilibrium (PBE), which parallels the notion of the subgame perfect equilibrium (SPE) in a multistage game with complete information and can be considered as a refinement of the Bayesian NE (BNE) in a multistage game with incomplete information. To achieve the PBE, players' behaviors must be sequentially rational [10] defined as follows.

Definition 1: [10, p. 331-333] (Sequentially Rational) For each player $x$ with type $\theta_{x}$, and history $h^{t}$ at stage $t$, the player $x$ is said to be sequentially rational if choosing the action

$$
\overline{\mathbf{a}}_{x}^{t}\left(\theta_{x}\right)=\arg \max _{\mathbf{a}_{x}^{t} \in \mathcal{A}_{x}} \sum_{\boldsymbol{\theta}_{-x}} \mu_{x}\left(\boldsymbol{\theta}_{-x} \mid h^{t}\right) \mathcal{P}_{x}\left(\mathbf{a}_{x}^{t}, \overline{\mathbf{a}}_{-x}^{t}\left(\boldsymbol{\theta}_{-x}\right)\right)
$$


for discrete set $\boldsymbol{\theta}$, where $\overline{\mathbf{a}}_{x}^{t}$ and $\overline{\mathbf{a}}_{-x}^{t}$ denote the PBE action profiles with respect to player $x$ and all the players excluding $x$, respectively.

In the multistage Bayesian game, each player updates its beliefs on other players' types at each stage. The belief update from $\mu_{x}\left(\theta_{y} \mid h^{t}\right)$ to $\mu_{x}\left(\theta_{y} \mid h^{t+1}\right)$ can be obtained by the Bayes' rule. Specifically, for all $x, y, h^{t}$, and $a_{y}^{t} \in \mathcal{A}_{y}\left(h^{t}\right)$, if there exists $\breve{\theta}_{y}$ with $\mu_{x}\left(\breve{\theta}_{y} \mid h^{t}\right)>0$ and $\overline{\mathbf{a}}_{y}^{t}\left(\breve{\theta}_{y}\right)=\overline{\mathbf{a}}_{y}^{t}\left(\widehat{\theta}_{y}\right)$, then for all $\theta_{y}$ we have [10, p. 332]

$$
\begin{aligned}
\mu_{x}\left(\theta_{y} \mid h^{t+1}\right) & =\mu_{x}\left(\theta_{y} \mid h^{t}, \mathbf{a}^{t}\right)=\frac{\mu_{x}\left(\theta_{y} \mid h^{t}\right) p\left(\mathbf{a}^{t} \mid h^{t}, \theta_{y}\right)}{p\left(\mathbf{a}^{t} \mid h^{t}\right)} \\
& =\frac{\mu_{x}\left(\theta_{y} \mid h^{t}\right) p\left(\mathbf{a}_{y}^{t} \mid h^{t}, \theta_{y}\right)}{\sum_{\tilde{\theta}_{y}} \mu_{x}\left(\tilde{\theta}_{y} \mid h^{t}\right) p\left(\mathbf{a}_{y}^{t} \mid h^{t}, \tilde{\theta}_{y}\right)}
\end{aligned}
$$

where $p\left(\mathbf{a}_{y}^{t} \mid h^{t}, \theta_{y}\right)=\delta\left(\mathbf{a}_{y}^{t}\left(\theta_{y}\right)-\overline{\mathbf{a}}_{y}^{t}\left(\widehat{\theta_{y}}\right)\right)$ is the probability of $\mathbf{a}_{y}^{t}$ given $h^{t}$ and $\theta_{y}$ with $\delta(\cdot)$ being the Kronecker delta function.

\section{Perfect Bayesian Equilibrium and Joint KKT CONDITIONS}

Here, we establish a model for the profit functions of all players and describe how to obtain the perfect Baysian equilibrium using the joint KKT conditions. In the following, the stage index $\tau$ and the conditioning on history $h^{t}$ will be omitted in occasions without ambiguity.

\section{A. Utility Model}

As mentioned in Sec. II, the $i$ th PS leases bandwidth $b_{j i}$ to the $j$ th SS. The amount of $b_{j i}$ affects the remaining available bandwidth and consequently the QoS satisfaction of the $i$ th PS which is represented by the utility function $u_{l_{i}}\left(\mathbf{b}_{i}^{l} \mid \boldsymbol{\theta}, h^{t}\right)$. Then, the monetary profit of trading is $\sum_{j} p_{j i} b_{j i}=\left(\mathbf{p}_{i}^{l}\right)^{T} \mathbf{b}_{i}^{p}$. As a result, the total profit of the $i$ th PS is given by

$$
\mathcal{P}_{l_{i}}\left(\mathbf{p}_{i}^{l}, \mathbf{b}_{i}^{l} \mid \boldsymbol{\theta}\right)=u_{l_{i}}\left(\mathbf{b}_{i}^{l} \mid \boldsymbol{\theta}\right)+\left(\mathbf{p}_{i}^{l}\right)^{T} \mathbf{b}_{i}^{l} .
$$

A discrete set $\boldsymbol{\theta}$ is assumed in the formulation. Likewise, the total profit of the $j$ th SS is given by

$$
\mathcal{P}_{s_{j}}\left(\mathbf{p}_{j}^{s}, \mathbf{b}_{j}^{s} \mid \boldsymbol{\theta}\right)=u_{s_{j}}\left(\mathbf{b}_{j}^{s} \mid \boldsymbol{\theta}\right)-\left(\mathbf{p}_{j}^{s}\right)^{T} \mathbf{b}_{j}^{s}
$$

where the satisfaction of the $j$ th SS is represented by the utility function $u_{s_{j}}\left(\mathbf{b}_{j}^{s} \mid \boldsymbol{\theta}\right)$. In Sec. IV, we will present an example that models the utility functions $u_{l_{i}}\left(\mathbf{b}_{i}^{l} \mid \boldsymbol{\theta}\right)$ and $u_{s_{j}}\left(\mathbf{b}_{j}^{s} \mid \boldsymbol{\theta}\right)$ as concave functions.

\section{B. Self-Optimization and KKT Translation}

Since the $j$ th SS is a follower of the game, it can observe the sellers' action $\mathbf{p}_{j}^{s}\left(\hat{\boldsymbol{\theta}}_{l}\right)=\left(p_{j 1}\left(\hat{\theta}_{l_{1}}\right) \ldots p_{j N}\left(\hat{\theta}_{l_{N}}\right)\right)^{T}$. Note that $\mathbf{p}_{j}^{s}\left(\hat{\boldsymbol{\theta}}_{l}\right)$ is the optimal price corresponding to type profile $\hat{\boldsymbol{\theta}}_{l}$ and is observed by the $j$ th SS without knowledge of $\hat{\boldsymbol{\theta}}_{l}$. Due to the randomness of $\boldsymbol{\theta}_{s_{-j}}$ and $\boldsymbol{\theta}_{l}$, the optimal $\mathbf{b}_{j}^{s}$ of the $j$ th SS with type $\hat{\theta}_{s_{j}}$ is found with respect to the expected profit as follows:

$$
\overline{\mathbf{b}}_{j}^{s}=\arg \max _{\mathbf{b}_{j}^{s}} \mathbf{E}_{\boldsymbol{\theta}_{-s_{j}}, \boldsymbol{\theta}_{l}}\left\{\mathcal{P}_{s_{j}}\left(\mathbf{p}_{j}^{s}\left(\hat{\boldsymbol{\theta}}_{l}\right), \mathbf{b}_{j}^{s}\right)\right\} .
$$

The KKT condition [11] for the expected profit maximization of the $j$ th SS of type $\hat{\theta}_{s_{j}}$ is

$$
\left.\nabla_{\mathbf{b}_{j}^{s}} \mathbf{E}_{\boldsymbol{\theta}_{-s_{j}}, \boldsymbol{\theta}_{l}}\left\{\mathcal{P}_{s_{j}}\left(\mathbf{p}_{j}^{s}\left(\hat{\boldsymbol{\theta}}_{l}\right), \mathbf{b}_{j}^{s}\right)\right\}\right|_{\mathbf{b}_{j}^{s}=\overline{\mathbf{b}}_{j}^{s}}=\mathbf{0} .
$$

This, from (4), is equivalent to

$$
\left.\nabla_{\mathbf{b}_{j}^{s}} \mathbf{E}_{\boldsymbol{\theta}_{-s_{j}}, \boldsymbol{\theta}_{l}}\left\{u_{s_{j}}\left(\mathbf{b}_{j}^{s} \mid \hat{\theta}_{s_{j}}, \boldsymbol{\theta}_{-s_{j}}, \boldsymbol{\theta}_{l}\right)\right\}\right|_{\mathbf{b}_{j}^{s}=\overline{\mathbf{b}}_{j}^{s}}=\mathbf{p}_{j}^{s}\left(\hat{\boldsymbol{\theta}}_{l}\right) .
$$

We note that $\overline{\mathbf{b}}_{j}^{s}$ is a function of $\hat{\theta}_{s_{j}}$ and $\hat{\boldsymbol{\theta}}_{l}$, and thus we write $\overline{\mathbf{b}}_{j}^{s}\left(\hat{\theta}_{s_{j}}, \hat{\boldsymbol{\theta}}_{l}\right)$ to specify this dependency. It is also observed that the optimization problems for all SSs can be decoupled, i.e. the profit maximization for the $j$ th SS depends on all PSs and the $j$ th SS, but not on the other SSs. Hence, for each sequentially rational SS, it suffices to solve (5) or (6) without need to consider the actions of other SSs.

Next, we describe how each PS determines the PBE strategy. Since all PSs know the best demand $\overline{\mathbf{b}}_{j}^{s}\left(\hat{\theta}_{s_{j}}, \hat{\boldsymbol{\theta}}_{l}\right)$ of the $j$ th SS for all $j$, the KKT condition (6) for all SSs should be incorporated as constraints when each PS sets the bandwidth prices. Specifically, the $i$ th PS attempts to maximize its expected profit based on the beliefs $\mu\left(\boldsymbol{\theta}_{s}, \boldsymbol{\theta}_{-l_{i}} \mid h^{t}\right)$ about other players' private information, while considering the KKT conditions of SSs. The PBE strategy $\overline{\mathbf{p}}_{i}^{l}\left(\hat{\theta}_{l_{i}}\right)$ for the $i$ th PS with type $\widehat{\theta}_{p_{i}}$ is determined by

$$
\begin{aligned}
& \max _{\mathbf{p}_{i}^{l}} \mathbf{E}_{\boldsymbol{\theta}_{s}, \boldsymbol{\theta}_{-l_{i}}}\left\{\mathcal{P}_{l_{i}}\left(\mathbf{p}_{i}^{l}, \overline{\mathbf{b}}_{i}^{l}\left(\boldsymbol{\theta}_{s}, \hat{\theta}_{l_{i}}, \boldsymbol{\theta}_{-l_{i}}\right)\right)\right\} \\
& \text { s.t. } \quad \bar{b}_{j i}\left(\theta_{s_{j}}, \hat{\theta}_{l_{i}}, \boldsymbol{\theta}_{-l_{i}}\right) \geq 0, \quad \forall \boldsymbol{\theta}_{s}, \boldsymbol{\theta}_{-l_{i}} \\
& \sum_{j} \bar{b}_{j i}\left(\theta_{s_{j}}, \hat{\theta}_{l_{i}}, \boldsymbol{\theta}_{-l_{i}}\right) \leq W_{i}, \quad \forall \boldsymbol{\theta}_{s}, \boldsymbol{\theta}_{-l_{i}} \\
& \nabla_{\mathbf{b}_{j}^{s}} \mathbf{E}_{\boldsymbol{\theta}_{-s_{j}}, \boldsymbol{\theta}_{l}}\left\{u_{s_{j}}\left(\mathbf{b}_{j}^{s} \mid \boldsymbol{\theta}\right)\right\}=\mathbf{p}_{j}^{s}\left(\hat{\theta}_{l_{i}}, \boldsymbol{\theta}_{-l_{i}}\right), \forall \boldsymbol{\theta}_{s}, \boldsymbol{\theta}_{-l_{i}} .
\end{aligned}
$$

Constraints (8) and (9) ensure that the bandwidth demand to the $i$ th PS is realizable under all possible type profiles of other players. Constraint (10) corresponds to the KKT condition (6) for all SSs. In this work, we refer to the approach that translates the KKT condition in (6) into the constraint in (10) as KKT translation.

\section{Perfect Bayesian Equilibrium and Joint KKT Conditions}

From (1), the PBE at stage $t$ of the multistage Bayesian game is

$$
\overline{\mathbf{p}}_{i}^{l}\left(\theta_{l_{i}}\right)=\arg \max _{\mathbf{p}_{i}^{l}} \mathbf{E}_{\boldsymbol{\theta}_{s}, \boldsymbol{\theta}_{-l_{i}}}\left\{\mathcal{P}_{l_{i}}\left(\mathbf{p}_{i}^{l}, \overline{\mathbf{b}}_{i}^{l}\left(\boldsymbol{\theta}_{s}, \theta_{l_{i}}, \boldsymbol{\theta}_{-l_{i}}\right)\right)\right\}
$$

subject to (8)-(10). If constraint (10) is affine and the price profile $\overline{\mathbf{p}}_{-i}^{l}\left(\boldsymbol{\theta}_{-l_{i}}\right)$ for all type profiles is known, then the KKT condition is sufficient to solve the convex optimization problem. However, finding the PBE strategy profile $\overline{\mathbf{p}}_{-i}^{l}\left(\boldsymbol{\theta}_{-l_{i}}\right)$ for all possible $\boldsymbol{\theta}_{-l_{i}}$ requires the information of $\overline{\mathbf{p}}_{i}^{l}\left(\theta_{l_{i}}\right)$ for all possible $\theta_{l_{i}}$, which suggests that all PSs' KKT conditions have 
to be solved jointly. The joint KKT conditions are given by

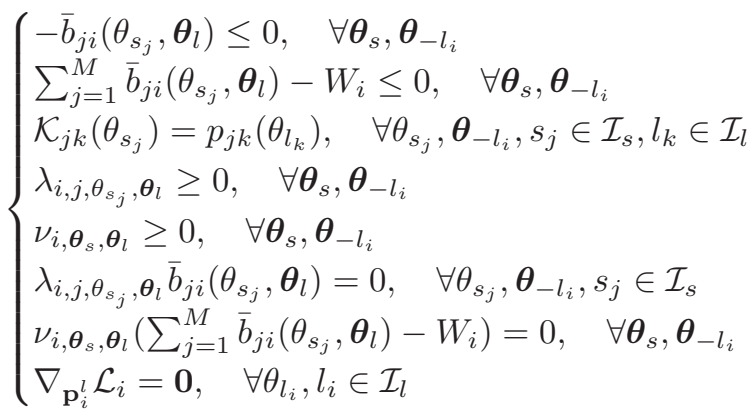

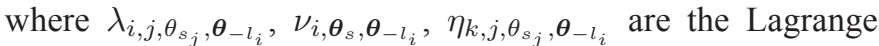
multipliers, $\mathcal{L}_{i}$ is the Lagrangian function of the $i$ th PS with type $\theta_{l_{i}}$, and

$$
\begin{gathered}
\mathcal{K}_{j k}\left(\theta_{s_{j}}\right)=\left.\frac{\partial}{\partial b_{j k}} \mathbf{E}_{\boldsymbol{\theta}_{-s_{j}}, \boldsymbol{\theta}_{l}}\left\{u_{s_{j}}\left(\mathbf{b}_{j}^{s} \mid \boldsymbol{\theta}\right)\right\}\right|_{\mathbf{b}_{j}^{s}=\overline{\mathbf{b}}_{j}^{s}\left(\theta_{s_{j}}, \boldsymbol{\theta}_{l}\right)}, \\
\forall l_{k} \in \mathcal{I}_{l} .
\end{gathered}
$$

The above joint KKT conditions can be solved using the active-set method [11], which provides the solution that specifies $\overline{\mathbf{p}}_{i}^{l}\left(\theta_{l_{i}}\right)$ for all possible $\theta_{l_{i}}$ and all $l_{i}$.

\section{Simulation Results}

\section{A. Simulation Setup}

In this section, we present an example of the multistage Bayesian game described in previous sections with specific utility functions. We extend the utility models in [4] to the system with multiple SSs and embed the private information in the utility functions. The profit function of the $i$ th PS is designed as

$$
\begin{aligned}
\mathcal{P}_{l_{i}}\left(\mathbf{p}_{i}^{l}, \mathbf{b}_{i}^{l}\right) & =\left(\mathbf{p}_{i}^{l}\right)^{T} \mathbf{b}_{i}^{l}+c_{1} \theta_{l_{i}} \\
& -c_{2} \theta_{l_{i}}\left(\mathrm{~B}_{i}^{\text {req }}-k_{i}^{(l)} \frac{W_{i}-\sum_{j=1}^{M} b_{j i}}{\theta_{l_{i}}}\right)^{2}
\end{aligned}
$$

where $c_{1}$ and $c_{2}$ are constant weights, $\mathrm{B}_{i}^{\text {req }}$ is the bandwidth requirement for a primary connection, $k_{i}^{(l)}=$ $\log _{2}\left(1+\frac{1.5 \gamma_{i}^{l}}{\ln \left(0.2 / \mathrm{BER}_{i}^{\mathrm{tar}}\right)}\right)$ denotes the spectral efficiency for the $i$ th PS with $\gamma_{i}^{l}$ being the signal-to-noise ratio (SNR) at the $i$ th PS's receivers and $\mathrm{BER}_{i}^{\mathrm{tar}}$ being the target bit-errorrate (BER). The private information $\theta_{l_{i}} \in \Theta_{l}$ (i.e., the type in the Bayesian game unknown to other players) represents the number of radio connections in the $i$ th PS. Note that the second term on the right-hand-side of (14) corresponds to the revenue of maintaining primary connections, and the third term quantifies the cost of sharing the spectrum with SSs.

The profit function of the $j$ th $\mathrm{SS}$ is designed as

$$
\begin{aligned}
& \mathcal{P}_{s_{j}}\left(\mathbf{p}_{j}^{s}, \mathbf{b}_{j}^{s}\right)=-\left(\mathbf{p}_{j}^{s}\right)^{T} \mathbf{b}_{j}^{s} \\
& +\frac{1}{\theta_{s_{j}}}\left[\sum_{i}^{N} b_{j i} k_{i}^{\left(s_{j}\right)}-\frac{1}{2}\left(\left(\mathbf{b}_{j}^{s}\right)^{T} \mathbf{b}_{j}^{s}+2 \xi_{j} \sum_{k \neq i} b_{j k} b_{j i}\right)\right]
\end{aligned}
$$

where $\xi_{j}$ is the $j$ th SS's spectrum substitutability as defined in [4], and $k_{i}^{\left(s_{j}\right)}=\log _{2}\left(1+\frac{1.5 \gamma_{j i}^{s}}{\ln \left(0.2 / \mathrm{BER}_{j}^{\text {tar }}\right)}\right)$ denotes the the spectral efficiency required by the $j$ th $\mathrm{SS}$ on the spectrum owned by the $i$ th PS with $\gamma_{j i}^{s}$ being the SNR at the $j$ th SS's receiver. Compared with the utility in [4], we introduce the private information $\theta_{s_{j}}$ as a weighting factor to the QoS term in (15). This private information is related to the number of active CR connections in an SS. For example, if there is no $\mathrm{CR}$ connection in the $j$ th SS, it must have zero profit in terms of QoS and the corresponding $\theta_{s_{j}}$ is set to infinity.

In the following, we will show the effectiveness of the proposed joint KKT method. The type space of PS is set as $\Theta_{P}=\{10,11,12\}$ and the type space of SS $\Theta_{S}=\{1,2,3\}$. The initial beliefs are assumed uniform over the type space, i.e. $\mu\left(\theta_{l_{i}} \mid h^{0}\right)=\frac{1}{3}$ for all $l_{i}$ and $\mu\left(\theta_{s_{j}} \mid h^{0}\right)=\frac{1}{3}$ for all $s_{j}$. We set $c_{1}=2$ and $c_{2}=2$ in (14), and $\xi_{j}=0.4$ for all $s_{j}$ in (15).

\section{B. Numerical Results and Discussion}

We present the evolution of action profile over stages of the proposed multistage spectrum trading game with two SSs and two PSs for two different cases (Case 1 and Case 2), and compare the results with those in [4] that correspond to the unconstrained (denoted "unc" in simulation figures) spectrum sharing with single SS and multiple PSs.

1) Case 1: We first study the behavior of the sequence of equilibrium strategies of the proposed multistage game, under $W_{1}=15 \mathrm{MHz}$ and $W_{2}=15 \mathrm{MHz}, \hat{\theta}_{l_{1}}=10, \hat{\theta}_{l_{2}}=10$, $\hat{\theta}_{s_{1}}=1, \hat{\theta}_{s_{2}}=2, \gamma_{1}^{l}=15, \gamma_{2}^{l}=15, \gamma_{11}^{s}=22, \gamma_{12}^{s}=18$, $\gamma_{21}^{s}=18, \gamma_{22}^{s}=22, \mathrm{~B}_{1}^{\text {req }}=2 \mathrm{Mbps}$ and $\mathrm{B}_{2}^{\text {req }}=2 \mathrm{Mbps}$. Fig. 3 shows the equilibrium pricing and the equilibrium bandwidth demands. It is observed that, since $\gamma_{11}^{s}>\gamma_{12}^{s}$, SS1 demands more bandwidth from PS1 than from PS2. Consequently, PS1 sets higher price to SS1 than PS2 does. On the other hand, SS2 demands more from PS2 than from PS1 since $\gamma_{22}^{s}>\gamma_{21}^{s}$. Therefore, PS2 sets higher price to SS2 than PS1 does. Note that, while $\gamma_{22}^{s}>\gamma_{12}^{s}$, we observe that PS2 sets lower price to SS2 than to SS1. This is because SS2 has private type $\hat{\theta}_{s}=2$ and therefore, from (15), puts less emphasis on the QoS satisfaction, or equivalently, is more concerned with the monetary expense. Thus, both PSs set lower price to SS2 to stimulate the demand.

We also observe from Fig. 3 that the spectrum trading game without considering the bandwidth constraints evolve to the same bandwidth pricings and demands as that which considers the joint KKT conditions after the belief updates converge. This is because the solutions are strictly feasible with the simulation settings for Case 1 .

2) Case 2: Next, we consider the following setting: $W_{1}=$ $15 \mathrm{MHz}$ and $W_{2}=15 \mathrm{MHz}, \hat{\theta}_{l_{1}}=10, \hat{\theta}_{l_{2}}=12, \hat{\theta}_{s_{1}}=1$, $\hat{\theta}_{s_{2}}=2, \gamma_{1}^{l}=15, \gamma_{2}^{l}=15, \gamma_{11}^{s}=22, \gamma_{12}^{s}=22, \gamma_{21}^{s}=22$, $\gamma_{22}^{s}=22, \mathrm{~B}_{1}^{\text {req }}=2 \mathrm{Mbps}$ and $\mathrm{B}_{2}^{\text {req }}=2 \mathrm{Mbps}$.

The equilibrium pricings and the equilibrium demands are shown in Fig. 4. Each PS asks lower price to SS2 than to SS1, since SS2 with $\hat{\theta}_{s}=2$ puts less emphasis on the QoS satisfaction. The cost of sharing spectrum for PS2 is higher than that for PS1, since PS2 with a higher volume of local connections is less willing to share the spectrum in order to fulfill its primary users' QoS satisfaction. Consequently, PS2 would set a higher price, resulting in lower $b_{12}$ and $b_{22}$. Under 

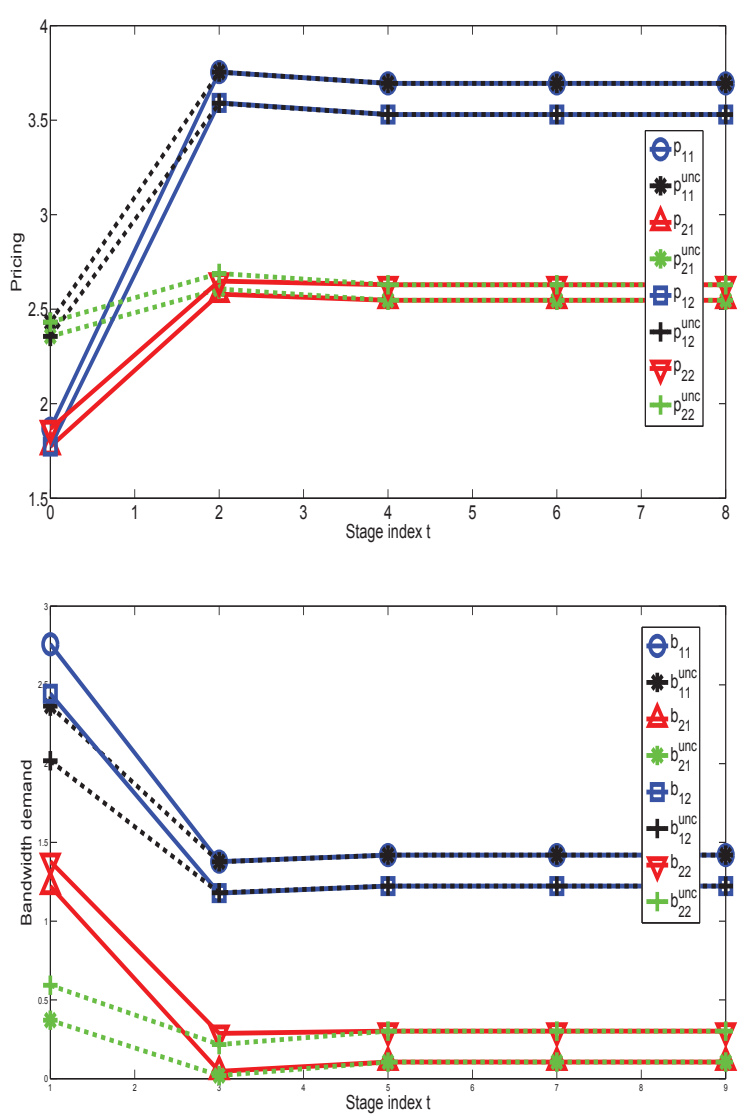

Fig. 3. The equilibrium strategies of PSs and SSs for Case 1.

this circumstance, SS1 and SS2 demand more $b_{11}$ and $b_{21}$, respectively, to compensate for $b_{12}$ and $b_{22}$. Moreover, with the setting in Case 2, the unconstrained demand $b_{22}^{\text {unc }}$ would be negative even when the beliefs converge correctly, while the proposed multistage game is always feasible.

\section{CONClusion}

We have studied a multistage Bayesian game with incomplete information of players in a sequential manner for spectrum trading in a CR network. To ensure that the trading is feasible, we have constrained the trading bandwidth for each player. In addition, we have proposed using the KKT translation and joint KKT conditions to solve the sequential optimization problem and yield the PBE at each stage. Finally, we have demonstrated the effectiveness of the joint KKT conditions, numerically studied the convergence of action profiles, and discussed how different parameters affect the equilibrium strategies in the simulations.

\section{REFERENCES}

[1] S. Haykin, "Cognitive radio: brain-empowered wireless communications," IEEE J. Select. Areas Commun., vol. 23, no. 2, pp. 201-220, Feb. 2005.

[2] Z. Ji and K. J. R. Liu, "Dynamic spectrum sharing: a game theoretic overview," IEEE Commun. Mag., pp. 88-94, May 2007.
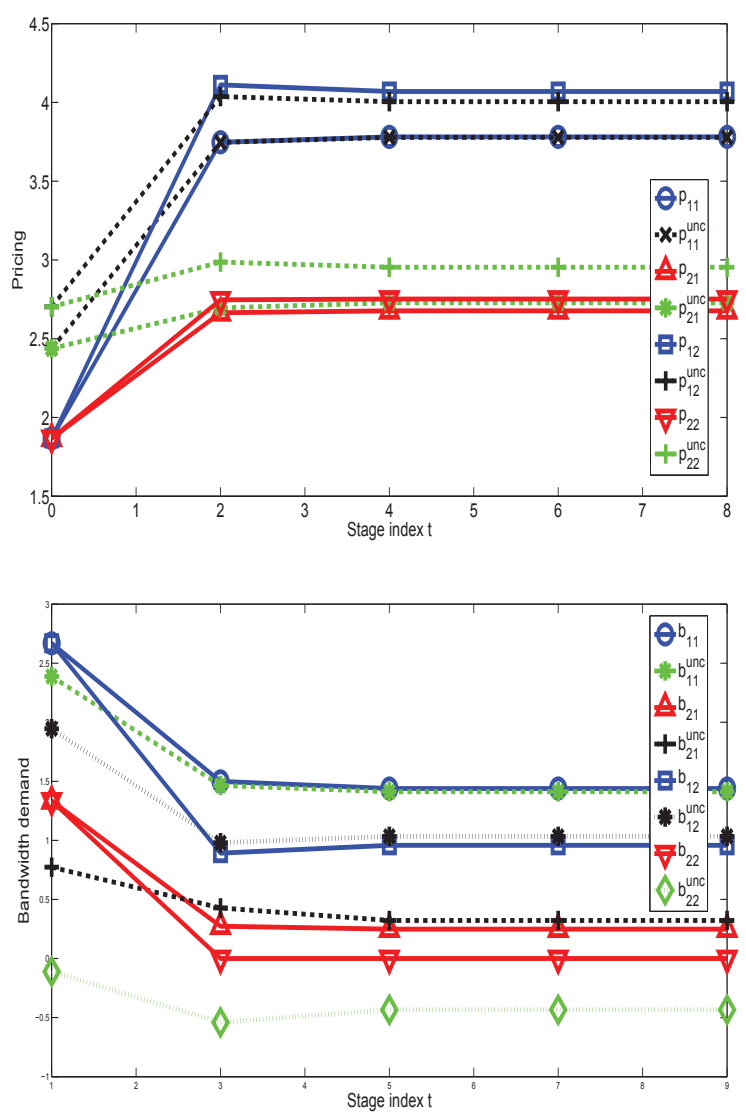

Fig. 4. The equilibrium strategies of PSs and SSs for Case 2.

[3] Z. Ji and K. J. R. Liu, "Multi-stage pricing game for collusion-resistant dynamic spectrum allocation," IEEE J. Select. Areas Commun., vol. 26, no. 1, pp. 182-191, Jan. 2008.

[4] D. Niyato and E. Hossain, "Competitive pricing for spectrum sharing in cognitive radio networks: dynamic game, inefficiency of Nash equilibrium, and collusion," IEEE J. Select. Areas Commun., vol. 26, no. 1, pp. 192-202, Jan. 2008.

[5] X. Feng, Q. Zhang, and B. Li, "HEAD: A hybrid spectrum trading framework for QoS-aware secondary users," in Proc. IEEE DYSPAN, Apr. 2014, pp. 489-497.

[6] J. Yin, G. Sun, and X. Wang, "Spectrum trading in cognitive radio networks: A two-stage market based on contract and Stackelberg game," in Proc. IEEE WCNC, Apr. 2013, pp. 1679-1684.

[7] P. Lin, X. Feng, and Q. Zhang, "Flexauc: Serving dynamic demands in spectrum trading markets with flexible auction," in Proc. IEEE INFOCOM, Mar. 2014, pp. 2265-2273.

[8] M. van der Schaar and F. Fu, "Spectrum access games and strategic learning in cognitive radio networks for delay-critical applications," Proceeding of the IEEE, vol. 97, no. 4, pp. 720-740, Apr. 2009.

[9] H. Jin, G. Sun, X. Wang, and Q. Zhang, "Spectrum trading with insurance in cognitive radio networks," in Proc. IEEE INFOCOM, Mar. 2012, pp. 2041-2049.

[10] D. Fudenberg and J. Tirole, Game Theory, The MIT Press, 1991.

[11] S. Boyd and L. Vandenberghe, Convex Optimization, CAMBRIDGE UNIVERSITY PRESS, 2004. 